\title{
APPENDIX IX
}

\section{Major officials of the Dutch East Indies Company, the British interim administration and the Netherlands Indies government 1780-1856 ${ }^{1}$}

Governors-general, 1780-1855

Willem Arnold Alting

Pieter Gerardus van Overstraten

Johannes Siberg

Albert Henricus Wiese

Herman Willem Daendels

Jan Willem Janssens

Gilbert Elliott Lord Minto ${ }^{2}$

Thomas Stamford Raffles ${ }^{3}$

John Fendall

Godert Alexander Gerard Philip

baron van der Capellen ${ }^{4}$

Leonard Pierre Joseph burggraaf

du Bus de Gisignies

Johannes van den Bosch
1780-2 November 1796

2 November 1796-22 August 1801

22 August 1801-15 June 1805

15 June 1805-14 January 1808

14 January 1808-16 May 1811

16 May-18 September 1811

6 August-19 October 1811

11 September 1811-12 March 1816

12 March-19 August 1816

19 August 1816-1 January 1826

4 February 1826-16 January 1830

16 January 1830-31 January $1834^{5}$

1 The following details are taken from Rouffaer 1905:601-2; De Haan 1935:477-681; Soekanto 1952:175-7; Poensen 1905:73-346, and Veth 1898, II:369-70, and many dates have also been checked against the available Residency letters. Wherever possible the dates given refer to the date of transfer of office rather than to the date of appointment.

2 Lord Minto was governor-general of India (1807-1813), the dates refer to the time he spent in Java during the conquest of the island by the British.

3 Both Raffles and his successor John Fendall held the rank of lieutenant-governor; the dates refer to Raffles' official appointment by Lord Minto and the day on which Fendall landed in Java.

$4 \quad$ Van der Capellen combined his post of governor-general with that of commissioner-general in the period 19-8-1816 to 16-1-1819. He was assisted as commissioner-general by two colleagues, C.Th. Elout and A.A. Buyskes. He handed over responsibility for the day-to-day administration of the Netherlands East Indies to Lieutenant-General Hendrik Merkus de Kock on 1-1-1826 and sailed for the Netherlands on 2-2-1826.

$5 \quad$ Van den Bosch combined the post of governor-general with that of commissioner-general in the period 1832-34, a post which gave him quasi-dictatorial powers to act on King William I's (r. 1813-1840) behalf at a time when the Cultivation System (1830-1870) was being implemented and the war against the Padris in West Sumatra was entering a crucial stage, see Stapel 1941:85. 
Jean Chrétien Baud

Dominique Jacques de Eerens

Pieter Merkus

Jan Jacob Rochussen

Albertus Jacobus Duymaer van Twist
31 January 1834-29 February $1836^{6}$

29 Feb 1836-30 May 1840 (died in post)

6 Jan 1841-2 August 1844 (died in post) ${ }^{7}$

30 September 1845-12 May 1851

12 May 1851-22 May 1856

Governors of Java's Northeast Coast, Semarang, 1780-1808

Johannes Siberg

Jan Greeve

Pieter Gerardus van Overstraten

Johan Frederik baron van Reede tot de Parkeler

Nicolaus Engelhard

Residents at Surakarta, 1790-1858

Johan Frederik baron van Reede tot de Parkeler

Barend Jan van Nieuwkerken, genaamd Nijvenheim

Johannes Gerardus van den Berg

Bogislaus Friedrich von Liebeherr

Jacob Andries van Braam (1)

Willem Nicolaas Servatius

(Assistant Resident)

Jacob Andries van Braam (2)

Lt. Col. Alexander Adams

Hugh Hope

Lt. Richard Hart (Assistant Resident)

Major Jeremiah Martin Johnson

Diederik Willem Pinket van Haak

Wouter Hendrik van IJsseldijk

Hendrik Ferdinand Lippe (1)
September 1780-September 1787

September 1787-1 September 1791

1 September 1791-2 November 1796

2 November 1796-6 March 1801

6 March 1801-13 May 1808

(dismissed by Daendels and the post abolished)

6 Baud had served as ad interim governor-general with responsibility for the oversight of day-to-day administration after his arrival in Java on 10-1-1833 to 31-1-1834 while Van den Bosch was serving as commissioner-general (see above note 5). He was confirmed in post following Van den Bosch's departure to assume the post of minister of the colonies (1834-1839), see Stapel 1941:85.

$7 \quad$ Merkus had been appointed ad interim governor-general on 3-10-1840 but could only take up his post in early 1841. He was confirmed in post on 14-2-1843. After his death on a sea voyage to Surabaya to restore his health, the vice-president of the Council of the Indies, Jonkheer J.C. Reynst, served as ad interim governor-general until the arrival of J.J. Rochussen on 29-9-1845, see Stapel 1941:93. 
Rijck van Prehn

Hendrik Ferdinand Lippe (2)

Huibert Gerard Nahuys van Burgst

(acting Resident)

Adriaan Mauritz Theodorus

Baron de Salis

Hendrik Mauritz MacGillavry (1)

(acting Resident)

Jan Izaäk van Sevenhoven

Hendrik Mauritz MacGillavry (2)

Mr. Huibert Gerard Nahuys van Burgst (2)

Mr. Johan Frederik Walraven van Nes

(acting Resident)

Hendrik Mauritz MacGillavry (3)

Frans Gerhardus Valck

(acting Resident)

J.F.T. Mayor

C.L. Hartmann

Mr. Willem Carel Emile Baron de Geer

Hendrik Frederik Buschkens

(acting Resident)

Residents at Yogyakarta, 1786-1855

Wouter Hendrik van IJsseldijk

Johannes Gerardus van den Berg

Matthijs Waterloo

Pieter Engelhard (1)

Gustaf Wilhelm Wiese

Johannes Wilhelmus Moorrees

Bartholomeus Jacobus Driessen

(acting Resident)

Pieter Engelhard (2)

John Crawfurd (1)

Lt. Arthur Aston Homer

(Assistant-Resident)

John Crawfurd (2)

Col. John Eales (acting Resident)

John Crawfurd (3)

Capt. Robert Clement Garnham

Lt. Edward Taylor (Assistant-Resident)

Dr. Daniel Ainslie

John Crawfurd (4)

Huibert Gerard Nahuys van Burgst (1)
19 January 1818-4 December 1819

(suspended)

4 December 1819-8 March 1820

8 March 1820-29 April 1822

29 April 1822-15 July 1823

15 July 1823-January 1824

January 1824-7 January 1825

7 January 1825-August 1827

(suspended)

August 1827-November 1830

Nov-Dec 1830

Dec 1830-March 1834

March-December 1834

December 1834-September 1843

September 1843-26 June 1846

26 June 1846-December 1850

March 1851-October 1858

(retired)

September 1786-January 1798

January 1798-26 July 1803

26 July 1803-14 April 1808

14 April-3 December 1808

3 December 1808-9 March 1810

9 March-24 August 1810

24 August-1 September 1810

1 September 1810-15 November 1811

15 November 1811-24 April 1813

24 April-December 1813

December 1813-12 April 1814

29 May-5 July 1814

5 July-31 August 1814

20 September 1814-31 August 1815

31 August-1 November 1815

1 November 1815-22 January 1816

22 January-14 August 1816

14 August-6 October 1816 
Johan Diederik Kruseman (provisional Resident)

Huibert Gerard Nahuys van Burgst (2)

Robbert Christiaan Nicolaas d'Abo

(Assistant Resident)

Huibert Gerard Nahuys van Burgst (3)

Adriaan Mauritz Theodore

Baron de Salis (acting Resident)

Anthonië Hendrik Smissaert

Hendrik Mauritz MacGillavry

(acting Resident)

Jan Izaäk van Sevenhoven

(acting Resident)

Pieter Herbert Baron van

Lawick van Pabst

Mr. Johan Frederik Walraven van Nes

(acting Resident)

Jan Izaäk van Sevenhoven

(acting Resident)

Mr. Johan Frederik Walraven van Nes (2)

Franciscus Gerardus Valck (1)

J.F.T. Mayor (acting Resident)

Frans Gerhardus Valck (2)

Arnold Adriaan Buyskes (acting Resident)

R. de Fillietaz Bousquet

Albert Hendrik Wendelin baron de Kock

J.J. Hasselman

Mr Willem Carel Emile Baron de Geer
6 October 1816-24 January 1817

24 January 1817-October $1818^{8}$

October 1818-5 May 1819

5 May 1819-1 November 1822

1 November 1822-11 February

1823

11 February 1823-26 September 1825

(dismissed)

26 September-28 October 1825

28 October 1825-10 March 1827

10 March-October 1827

October 1827-November 1830

November-December 1830

December 1830-March 1831

March 1831-5 July 1838

5 July-14 December 1838

14 December 1838-July 1841

July 1841-May 1845

May 1845-24 November 1848

(suspended)

28 December 1848-May 1851

8 July 1851-January 1855

Feb. 1855-6 May 1856 (died in post)

8 According to Rouffaer in Winter 1902:124 note 1, Nahuys van Burgst left Yogyakarta sometime between 10 and 16 October 1818 . 